\title{
Specification and Verification of Graph-Based Model Transformation Properties ${ }^{\star}$
}

\author{
Gehan M. K. Selim ${ }^{1}$, Levi Lúcio ${ }^{2}$, James R. Cordy ${ }^{1}$, Juergen Dingel ${ }^{1}$, and \\ Bentley J. Oakes ${ }^{2}$ \\ 1 School of Computing, Queen's University, Kingston ON K7L2N8, Canada, \\ \{gehan, cordy, dingel\}@cs.queensu.ca \\ 2 School of Computer Science, McGill University, Montreal QC H3A2A7, Canada, \\ levi@cs.mcgill.ca, bentley.oakes@mail.mcgill.ca
}

\begin{abstract}
We extend a previously proposed symbolic model transformation property prover for the DSLTrans transformation language. The original prover generated the set of path conditions (i.e., symbolic transformation executions), and verified atomic contracts (constraints on inputoutput model relations) on these path conditions. The prover evaluated atomic contracts to yield either true or false for the transformation when run on any input model. In this paper we extend the prover such that it can verify atomic contracts and more complex properties composed of atomic contracts. Besides demonstrating our prover on a simple transformation, we use it to verify different kinds of properties of an industrial transformation. Experiments on this transformation using our prover show a speed-up in verification run-time by two orders of magnitude over another verification tool that we evaluated in previous research.
\end{abstract}

Keywords: MDD, model transformation, verification, property prover.

\section{Introduction}

In Model-Driven Development (MDD), models are the basic blocks of software development, and model transformations are used to map between models conforming to different metamodels. Given their key role in MDD, verification of transformations is becoming of increasing interest to researchers $[2,16]$.

In this study, we formulate and focus on the following research question: "How can we efficiently verify properties of transformations expressed as input-output model relations?". We focus on properties expressed as input-output model relations since they have been highly investigated in the literature, using both textual (e.g., $[9,3])$ and graphical (e.g., $[11,20])$ property languages. After a thorough review of studies addressing the same research question, we found several limitations in the state of the art. For example, several studies translate either the transformation (e.g., $[9,10]$ ) or the property (e.g., [11]) of interest to an intermediate format to facilitate verification, without proving the soundness of the translation. Secondly, other studies propose incomplete verification techniques that do not account for all possible transformation executions (e.g., [9]). Finally,

* This work is supported in part by NSERC, as part of the NECSIS Automotive Partnership with General Motors, IBM Canada and Malina Software Corp. 
a large number of studies proposed input-dependent verification techniques [2] (e.g., Henshin [4], AGG [20]) that prove properties for transformations only when run on a specific input. More general, input-independent techniques are needed were property verification is to be performed only once for the transformation, and verification results are to be guaranteed for all possible inputs.

In an attempt to answer the above research question and overcome limitations of previous studies, we investigate verifying properties of transformations implemented in the graph-based model transformation language DSLTrans [7]. DSLTrans is non-Turing complete, i.e., DSLTrans cannot specify transformations that require unbounded loops (e.g., simulation transformations). We extend a symbolic model transformation property prover for DSLTrans $[14,12]$ that was previously limited to verifying atomic contracts (i.e., constraints on input-output model relations). The extension we present in this paper supports a more expressive property language that facilitates verifying atomic contracts and compositions of atomic contracts in the form of propositional logic formulae. Moreover, our prover now handles rules that overlap in their application.

The contribution of this study, at a high level, is extending a DSLTrans property prover that is input-independent [2], i.e., verification results generated by the prover hold for all possible inputs. Our specific contributions are:

- We describe how our prover currently handles overlapping rules (Section 4).

- We introduce our new property language, and show how it can be used to express commonly occurring properties, e.g., multiplicity invariants. (Section 5).

- We apply our extended prover to an industrial case study [18] (Section 6).

- We demonstrate how our extensions of the prover led to a two orders of magnitude improvement in execution time over the verification tool we used in another study [17]. We also discuss the strengths and limitations of our prover (Section 7).

This study adds to the state of the art (Section 8) and is useful to transformation verification research in general. We provide some evidence for our prover's scalability and usefulness since verification using our prover does not have to be performed for each input. Thus, we motivate researchers to adopt our prover. Moreover, users of languages other than DSLTrans can benefit from our study in two ways: (1) the study can be used as a guide to develop input-independent verification for any language; (2) higher order transformations (HOTs) can be developed to convert transformations in other languages to DSLTrans to enable using our prover. To develop such HOTs, research has to be conducted to understand what class of transformations can be translated to DSLTrans.

Section 2 summarizes DSLTrans and it's simplest properties; Section 3 overviews our prover's architecture; Section 4 describes path condition generation; Section 5 discusses our prover's verification technique; Section 6 demonstrates an industrial case study; Section 7 discusses our prover's strengths and limitations; Section 8 reviews related work; Section 9 concludes and presents future work.

\section{The DSLTrans Model Transformation Language}

DSLTrans [7] is a graph-based transformation language that can be used to specify out-place (i.e., input-preserving), model transformations that are confluent and terminating by construction. DSLTrans rules are constructive - elements 


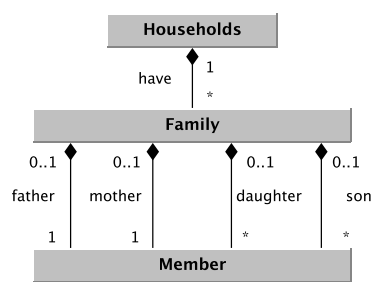

Fig. 1. Household Language

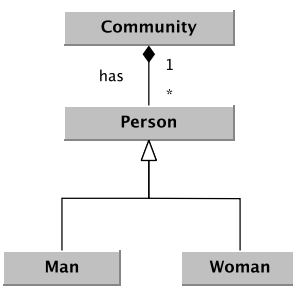

Fig. 2. Community Language

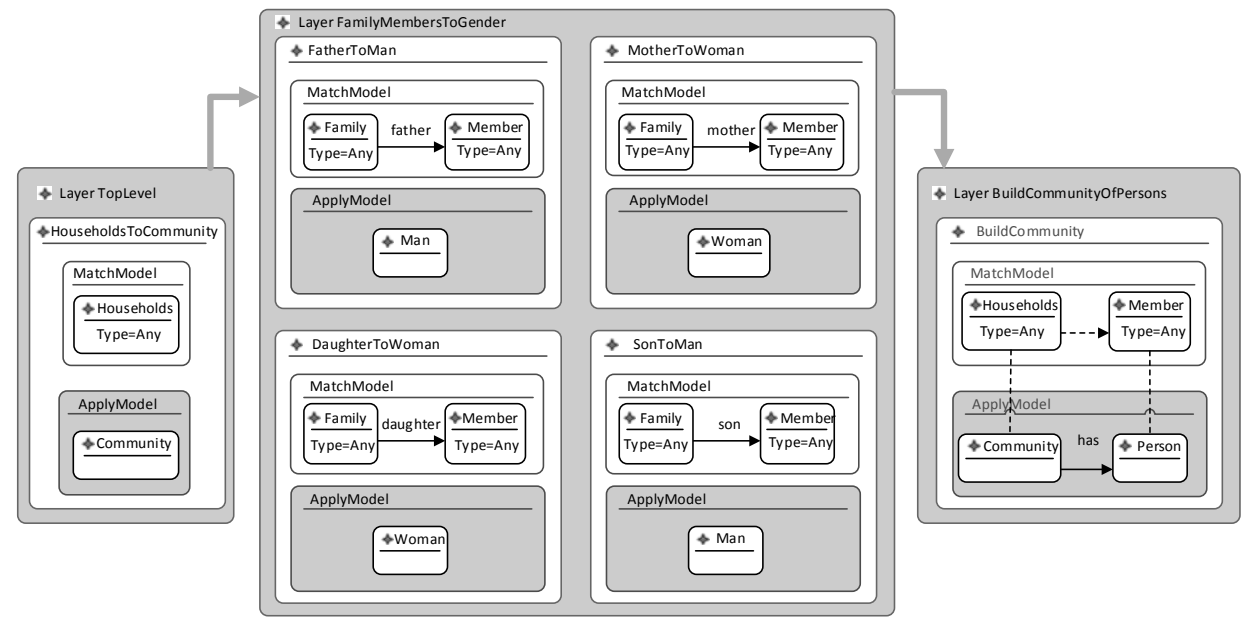

Fig. 3. The Persons Transformation expressed in DSLTrans.

can be created but not deleted. The semantics of DSLTrans (currently defined using set theory) are in-line with, and can be defined using, pushout approaches. We demonstrate DSLTrans using a simple transformation as a running example.

Figs. 1 and 2 present two metamodels used to describe different representations of a set of people. The 'Household Language' represents people as members of families which in turn form a set of households. The 'Community Language' represents people as men or women who belong to a community.

Fig. 3 presents a DSLTrans transformation that aims to transform family members in the 'Household Language' (source metamodel) into men and women of a community in the 'Community Language' (target metamodel). In what follows, we refer to the transformation in Fig. 3 as the Persons transformation.

A DSLTrans transformation is composed of an ordered set of layers (e.g., 'TopLevel', 'FamilyMembersToGender', and 'BuildCommunityOfPersons' layers in Fig. 3) that are executed sequentially. A layer consists of a set of transformation rules that execute in a non-deterministic order but produce a deterministic result. Each rule is a pair (MatchModel, ApplyModel) where MatchModel is a pattern of source metamodel elements and ApplyModel is a pattern of target metamodel elements. For example, the MatchModel of the 'HouseholdsToCommunity' rule in the 'TopLevel' layer (Fig. 3) has one 'Households' class from the 'Household Language' and the ApplyModel has one 'Community' class from the 
'Community Language'. This means that 'Households' input model elements will be transformed into 'Community' output model elements.

When a DSLTrans rule executes, traceability links are created between each element in the rule's MatchModel and each element in the ApplyModel. These are used to keep track of which output elements came from which input elements.

We describe some DSLTrans constructs that are used to build the MatchModel of a DSLTrans rule. More DSLTrans constructs can be found in $[7,12]$.

- Match Elements are variables typed by source metamodel classes that can assume as values instances of that class from the input model. An example of a match element is the 'Family' element in the 'FatherToMan' rule (Fig. 3). Match elements can be of two types: Any match elements are bound to all matching instances in the input model, and Exists match elements are bound to only one (deterministic) matching instance in the input. All match elements in Fig. 3 are of type Any.

- Attribute Conditions are conditions on the attributes of a match element.

- Direct Match Links are links between two match elements that are typed by labelled relations of the source metamodel. These links can assume as values links having the same label in the input model.

- Indirect Match Links represent a path of containment associations between the linked match elements. For example, an indirect match link appears in the 'BuildCommunity' rule as a horizontal, dashed arrow between match elements.

- Backward Links link elements of the MatchModel and the ApplyModel of a rule, e.g., backward links are used in the 'BuildCommunity' rule and are denoted as vertical, dashed lines. Backward links are used to refer to traceability links between input and output model elements that are generated by the rules of previous layers.

Similar constructs can be used to build a rule's ApplyModel, as shown in Fig. 3.

- Apply elements are variables typed by target metamodel classes and linked by apply links. Apply elements that are not connected by backward links create output elements of the same type each time the MatchModel is found in the input. Apply elements that are connected by backward links are handled differently, e.g., 'BuildCommunity' rule connects 'Community' and 'Person' output elements that were formerly created from 'Households' and 'Member' input elements with a 'has' link.

- Apply elements can have apply attributes that can be set from references to one or more attributes of match elements.

DSLTrans AtomicContracts: An AtomicContract is the simplest property expressible in our prover. Each AtomicContract is a pair (pre, post) that specifies a property of the form: "if the input model satisfies the precondition pre, then the output model should satisfy the postcondition post". A precondition is a constraint on the transformation's input in the form of a structural relation between input elements. A postcondition is a constraint on the transformation's output in the form of a structural relation between output elements. Preconditions and postconditions are expressed using the same constructs as rules. Postconditions may also have traceability links to link postcondition elements to precondition elements. This signifies that the property will only match an output element that was previously created from an input element. Figs. 4 and 5 demonstrate two AtomicContracts for the Persons transformation. Fig. 4 is interpreted as follows: "a mother and a father in a family will always be transformed to a woman 


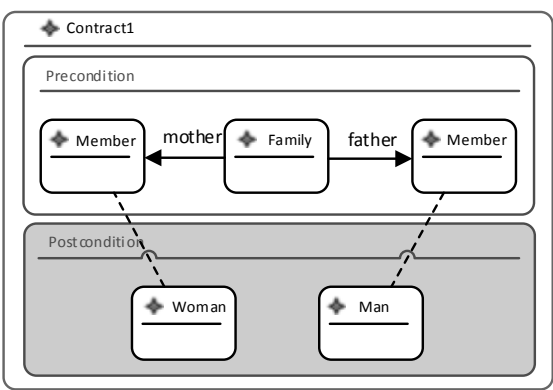

Fig. 4. Contract1; should hold.

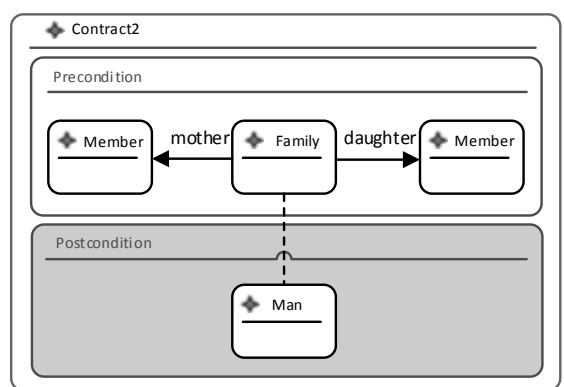

Fig. 5. Contract2; should not hold.

\begin{tabular}{|c|c|c|}
\hline $\begin{array}{c}\text { DSLTrans Transformation }+ \\
\text { Source \& Target Metamodels }\end{array}$ & Path Condition \\
Generation
\end{tabular}

Fig. 6. The architecture of our symbolic model transformation property prover.

and a man". Fig. 5 is interpreted as follows: "a family including a mother and a daughter will always be transformed to a man". Our prover should verify that Contract1 (Fig. 4) will always hold for the Persons transformation, while Contract2 (Fig. 5) will not always hold (with a counterexample).

\section{The Symbolic Model Transformation Property Prover}

Fig. 6 demonstrates our property prover's final architecture. Our prover takes four inputs: the DSLTrans transformation of interest, the transformation's source and target metamodels, and the property to verify. Verification is then carried out in two steps, as shown in Fig. 6. First, the prover generates the set of path conditions representing all possible executions of the input transformation (Section 4). Then, the prover verifies the input property on the generated set of path conditions and renders the property to be either true or false (with a counter example) for the transformation when run on any input model (Section 5).

We have chosen Python and T-Core [19] to implement our prover. T-Core is a Python library with primitives that support typed graph manipulation (e.g., graph matching/rewriting) and composition of these primitives into transformation blocks. The use of Python and T-Core allowed constructing our prover using MDD principles. In other words, all artifacts used at verification run-time are models (instances of explicit metamodels), all model-related computations are implemented as transformations, and all computations that do not directly manipulate models are implemented as Python algorithms that have been optimized to minimize memory usage and run-time. The models, metamodels, and transformations used at verification run-time are themselves automatically generated by higher order transformations in a compilation step that precedes verification.

\section{Generating the Set of Path Conditions}

Our prover generates a set of path conditions that symbolically represent the possible transformation executions. For a transformation with $n$ layers, our prover uses the transformation rules to build the path conditions in $n$ iterations. In 
Fig. 7, we demonstrate how the path conditions for the Persons transformation are generated in iterations. We identify every rule in each layer of Fig. 3 with a pair of numbers, e.g., $4_{2}$ corresponds to the fourth rule (ordered from top to bottom and then from left to right in Fig. 3) in the second layer (i.e., 'SonToMan' rule). We start off with the empty path condition, where we assume no transformation rule has been applied. To generate path conditions in iteration 1, the empty path condition is combined with all possible rule combinations of the first transformation layer. Similarly, to generate path conditions in iteration 2, each path condition from iteration 1 is combined with all applicable rule combinations of the second layer. A rule combination of the second layer that does not have backward links is always applicable, since it does not depend on rules from the first layer. Rule combinations of the second layer with backward links are combined with a path condition from iteration 1 only if the path condition generates the elements linked by backward links in the rule combination.

Each path condition thus accumulates a set of rules describing a possible path of rule applications through the transformation's layers. We refer to the accumulated MatchModels (or ApplyModels) of all the rules in a path condition as the path condition's match pattern (or apply pattern). Since our technique abstracts from how many times the rule executes for an input, a transformation rule only occurs once in each path condition. Thus, a path condition symbolically represents a set of concrete executions since each of the rules in a path condition can be concretely executed any number of times on an input model.

In Fig. 8, we show the path condition of the node with the dotted edge in Fig. 7. As shown from the numbers in the node, the path condition contains four combined rules (i.e., 'HouseholdsToCommunity', 'FatherToMan', 'MotherToWoman', 'BuildCommunity') and traceability links. When combining the rules, elements of the same type of the combined rules can be merged. This represents the fact that different rules may execute over the same input elements.

Only the path conditions from the last iteration are returned as the result since they capture all the possible complete transformation executions. Details on AtomicContracts and path condition generation can be found in [12].

Overlapping Rules: The industrial transformation presented later in Section 6 had overlapping rules which required treatment during path condition generation. Overlapping rules are defined as follows: when two rules in the same layer use match elements of the same metamodel classes of type Any or Exists, then the MatchModel of one rule syntactically subsumes the MatchModel of the other rule. For example, a rule having a MatchModel containing an Any match element of class 'A' is subsumed by a MatchModel of another rule that contains an Exists match element of class 'A' and an Any match element of class 'B'.

Our path condition generation algorithm was extended to handle overlapping rules. This extension led to a pronounced decrease in the number of generated path conditions in our case study, since a set of rules in a subsumption relation (described above) can often be merged into a smaller set of rules. Depending on whether rules overlap totally or partially, rule merge may be done before path condition generation or during path condition generation. For transforma- 


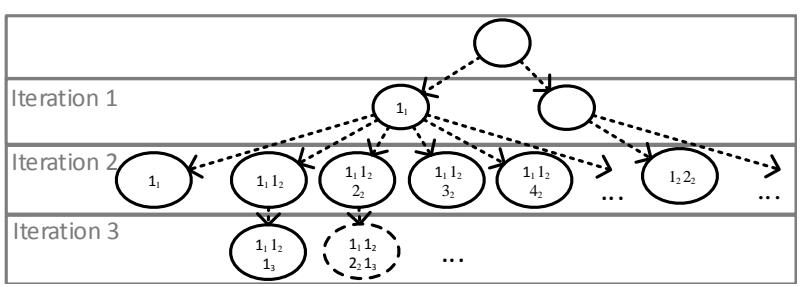

Fig. 7. Generation of the set of path conditions in iterations.

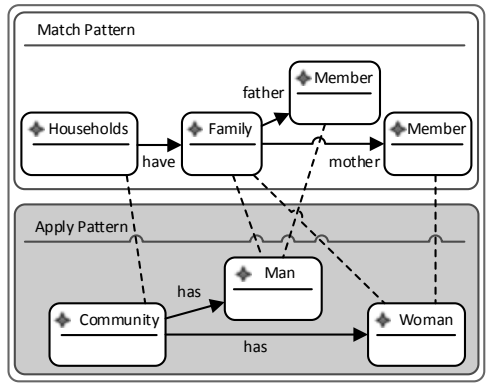

Fig. 8. A path condition of the Persons transformation.

tions with rule overlaps, this extension leads to an improved management of the combinatorial explosion in path condition generation.

\section{Verification of the Property of Interest}

We extended the technique proposed in [14] for verifying AtomicContracts of DSLTrans transformations to enable the verification of more complex properties. Our extended technique employs the following syntax and semantics.

Syntax: Our syntax is based on propositional logic. An AtomicContract (pre,post) is the smallest unit in our property language. A propositional formula can be built using one or more AtomicContracts and the operators $\neg_{t c}(n o t)$, $\vee_{t c}($ or $), \wedge_{t c}($ and $)$, and $\Longrightarrow_{t c}$ (implication), where $t c$ stands for "transformation contract". Assuming that (pre,post) is an element of the set of AtomicContracts $A C$, the syntax of formulae is:

$$
\varphi:=(\text { pre, post })\left|\neg_{t c} \varphi\right| \varphi \vee_{t c} \varphi\left|\varphi \wedge_{t c} \varphi\right| \varphi \Longrightarrow_{t c} \varphi
$$

Free variables can occur in any element $e$ of an AtomicContract's pre/ postcondition. This occurrence binds the free variable to all the matches found for $e$ within an instantiation of a MatchModel. Using the same free variable in different AtomicContracts allows these AtomicContracts to refer to the same matched element, e.g., Atomic Contract cont1 in Fig. 9 binds a matched element of type 'Community' to the free variable 'COMMUNITY' such that this element can be referred to in cont2 and cont3. The bindings of a set of free variables $\left\{v r_{1}, \ldots, v r_{n}\right\}$ (in elements $\left\{e_{1}, \ldots, e_{l}\right\}$ of an AtomicContract) to matched elements $\left\{m_{1}, \ldots, m_{n}\right\}$ in a path condition is expressed as a binding function $l=\left\{\left(\operatorname{var}_{1}, m_{1}\right), \ldots,\left(\operatorname{var}_{n}, m_{n}\right)\right\}$, i.e., $l \in \mathcal{P}(F V \times B E)$, where $F V$ and $B E$ are the sets of free variables and bound elements, and $\mathcal{P}$ is the power set operator.

Semantics: We define a function $\operatorname{eval}_{\text {Atomic }}(p c, c)$ that evaluates an Atom$i c$ Contract $c=($ pre,post $)$ for a path condition $p c$ as follows: 
1. If $p c$ contains an isomorphic copy of pre but does not contain an isomorphic copy of post, then $\operatorname{eval}_{\text {Atomic }}(p c, c)$ returns false (i.e., $c$ does not hold for $p c$ and the transformation) and an empty set of binding functions $L=\emptyset$.

2. Otherwise, $e^{2} \operatorname{lal}_{\text {Atomic }}(p c, c)$ returns true (i.e., $c$ holds for $p c$ ) and a set of binding functions $L$ for the free variables of $c$, where $L \subseteq \mathcal{P}(F V \times B E)$.

Thus, eval Atomic is defined as eval $_{\text {Atomic }}: P C \times A C \rightarrow\{$ true, false $\} \times \mathcal{P}(F V \times$ $B E$ ), where $P C$ is the set of path conditions of a transformation $\tau$. Note that a set $L$ of binding functions is returned since an AtomicContract may evaluate to true using different bindings of the free variables. Thus, $L$ is constructed from all binding functions $l_{i}$ returned by all possible subgraph isomorphisms.

Assuming that FORMULAE is the set of elements generated by the grammar in Eqn.(1), we evaluate a formula $\varphi$ for a path condition $p c \in P C$ using a function eval:PC $\times$ FORMULAE $\rightarrow\{$ true, false $\} \times \mathcal{P}(F V \times B E)$ as follows:

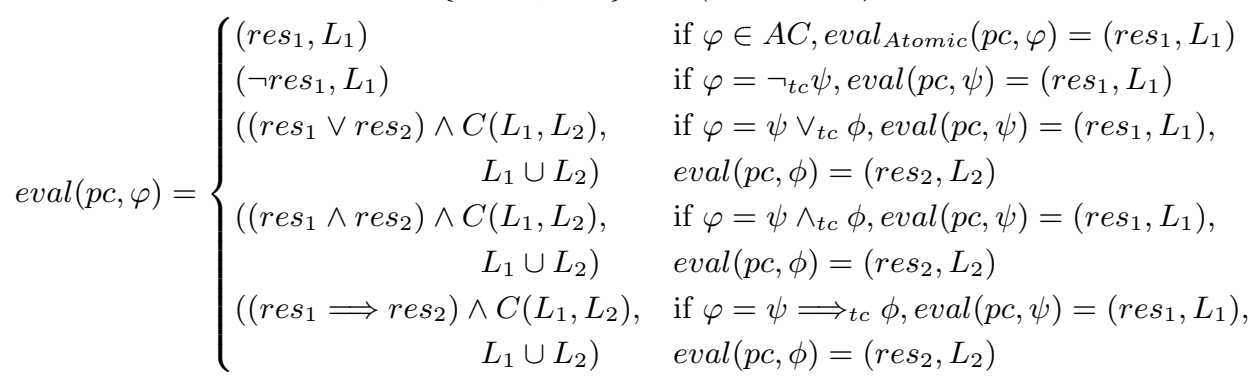

where the semantics of the propositional operators $(\neg, \vee, \wedge, \Longrightarrow)$ is standard, and res $_{i} \in\{$ true, false $\}$. The consistency function $C: \mathcal{P}(F V \times B E) \times \mathcal{P}(F V \times B E) \rightarrow$ $\{$ true, false $\}$ checks for two sets of binding functions (e.g., $L$ and $\left.L^{\prime}\right)$ that all free variables bound by a binding function in the first set $L$ will always be bound to the same elements by a binding function of the second set $L$ ' as follows:

$$
\begin{aligned}
C\left(L, L^{\prime}\right)= & \forall l \in L, \exists l^{\prime} \in L^{\prime}:\left(\forall v \in F V_{l}:\left((v, m) \in l \wedge\left(v, m^{\prime}\right) \in l^{\prime}\right) \Longrightarrow m=m^{\prime}\right) \text { and } \\
& \forall l^{\prime} \in L^{\prime}, \exists l \in L:\left(\forall v \in F V_{l^{\prime}}:\left(\left(v, m^{\prime}\right) \in l^{\prime} \wedge(v, m) \in l\right) \Longrightarrow m^{\prime}=m\right)
\end{aligned}
$$

where $m, m^{\prime} \in B E$, and $F V_{l}, F V_{l^{\prime}}$ are the sets of free variables used in $l$ and $l$ ' respectively. Based on the former definitions, we evaluate a formula $\varphi$ for a transformation $\tau$ (with path conditions $P C$ ) using a function $\operatorname{eval}(\tau, \varphi)$ :

$$
\operatorname{eval}(\tau, \varphi)= \begin{cases}\text { true } & \text { if } \forall p c \in P C: \operatorname{eval}(p c, \varphi)=(\text { true }, L) \\ \text { false } & \text { otherwise }\end{cases}
$$

where $\mathrm{L}$ is any set of binding functions. Thus, $\operatorname{eval}(\tau, \varphi)$ renders a property $\varphi$ to be true or false for a transformation $\tau$ by verifying $\varphi$ for each path condition. Function $\operatorname{eval}(\tau, \varphi)$ returns true only if for all path conditions of $\tau, \varphi$ holds and the bindings of all free variables consistently refer to the same elements.

Formulae of AtomicContracts: The new syntax and semantics allows us to formulate complex properties by composing propositional formulae of AtomicContracts. We demonstrate how the AtomicContracts in Fig. 9 (i.e., cont1, cont2, cont3) together with free variables can be used with different proposi- 

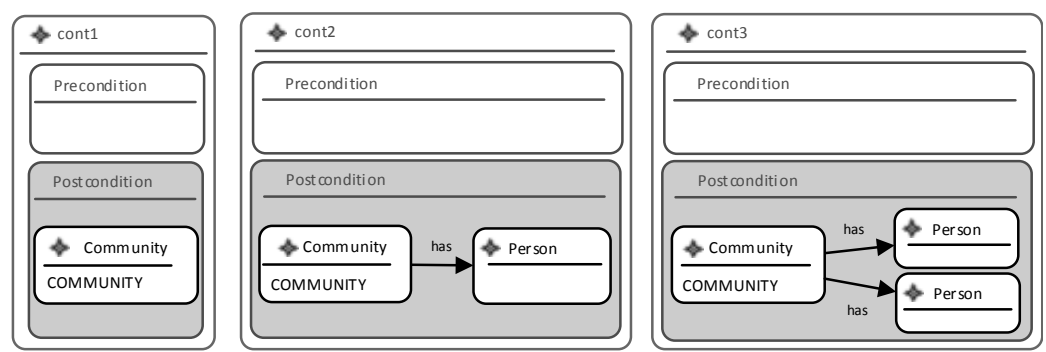

Fig. 9. Three Atomic Contracts that can be used with different propositional operators to convey different properties for the Persons transformation.

tional operators to convey multiplicity invariants ${ }^{1}$. A property that mandates that the Persons transformation will always generate an output where every community has one or more 'Persons' (i.e., a multiplicity invariant of '1...') can be expressed as 'cont $1 \Longrightarrow_{t c}$ cont 2 '. In other words, if an element of type 'Community' is generated in the output, then this element must have at least one 'Person'. Whereas the property 'cont $1 \Longrightarrow_{t c}\left(\operatorname{cont} 2 \wedge_{t c} \neg_{t c}\right.$ cont 3$)$ ' expresses a multiplicity invariant of '1..1' (i.e, if a 'Community' is generated in the output, then this 'Community' must have one 'Person' and not more).

\section{Industrial Case Study}

Previously in [18], we developed an industrial transformation that maps between subsets of a legacy metamodel for General Motors (GM) and the AUTOSAR metamodel. In that work, we focused on subsets of the metamodels that represent the deployment and interaction of software components. Later in [17], we proposed properties of interest for our GM-2-AUTOSAR transformation.

We use our prover to verify the properties proposed in [17] on the GM-2AUTOSAR transformation [18] after reimplementing it in DSLTrans. In this section, we summarize the transformation [18] and its properties [17]. Then, we discuss formulating and verifying these properties using our prover.

\subsection{GM-2-AUTOSAR Model Transformation}

The Source GM Metamodel: Fig. 10 illustrates the subset of the GM metamodel used in our transformation in $[18]^{2}$. A PhysicalNode may contain multiple Partitions (i.e., processing units). Multiple Modules can be deployed on a single Partition. A Module is an atomic, deployable, and reusable software element and can contain multiple Schedulers. A Scheduler is the basic unit for software scheduling. It contains behavior-encapsulating entities, and is responsible for providing/requiring Services to/from these behavior-encapsulating entities.

The Target AUTOSAR Metamodel: In AUTOSAR, an Electronic Component Unit (ECU) is a physical unit on which software is deployed. Fig. 11 shows the subset of the AUTOSAR metamodel [1] used by our transformation. The

\footnotetext{
${ }^{1}$ Note that the three AtomicContracts in Fig. 9 have empty preconditions meaning that they will match on any input model.

${ }^{2}$ We follow the same obfuscated naming conventions that we used for the GM metamodel in [18] for reasons of confidentiality.
} 


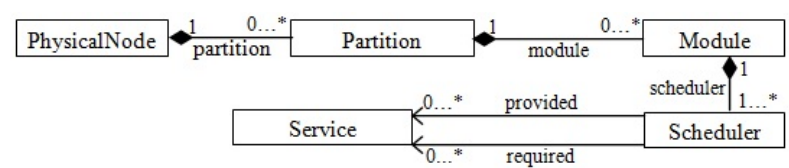

Fig. 10. Subset of the GM metamodel used by our transformation.

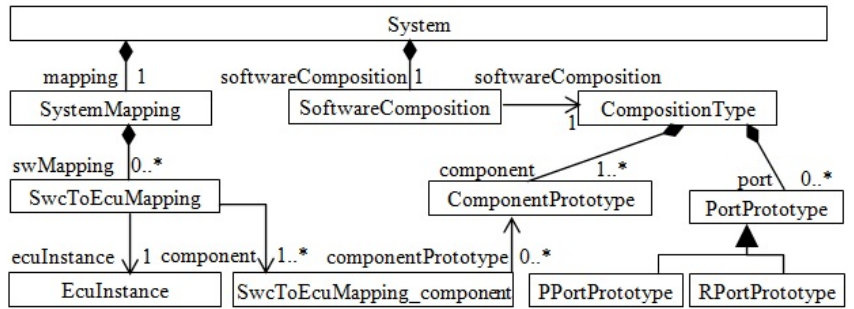

Fig. 11. Subset of the AUTOSAR metamodel used by our transformation.

ECU configuration is modeled using a System that aggregates SoftwareComposition and SystemMapping. SoftwareComposition points to CompositionType which eliminates any nested software components in a SoftwareComposition. SoftwareComposition models the architecture of the software components (i.e., ComponentPrototypes) deployed on an ECU and their ports (i.e., PPortPrototype/ RPortPrototype for providing/ requiring data and services).

SystemMapping binds software components to ECUs using SwcToEcuMappings. SwcToEcuMappings assign SwcToEcuMapping_components to an EcuInstance. SwcToEcuMapping_components, in turn, refer to ComponentPrototypes.

Reimplementation of the GM-2-AUTOSAR Transformation in DSLTrans: We reimplemented the GM-2-AUTOSAR transformation [18] in DSLTrans so that we can verify it in our prover. Table 1 shows the rules in each transformation layer, and the input/output types that are mapped/generated by each rule. Rules of the first and third layers create output elements. Rules of the second layer generate associations between elements created by the the first layer (shown in the actual transformation using backward links). Thus, the input and output types shown for the rules of the second layer are types that have already been matched and created and for which the rules create associations.

To represent positive application conditions (PACs) in our transformation rules, we use a combination of Any and Exists match elements (Section 2). For example, rule 'MapPhysNode2FiveElements' in Table 1 maps every PhysicalNode to five elements, only if the PhysicalNode is eventually connected to at least one Module. Thus, the MatchModel of rule 'MapPhysNode2FiveElements' has a PhysicalNode (Any) match element connected to Partition and Module (Exists) match elements. Similarly, rule 'MapModule' maps every Module (represented as Any match element) only if it is contained in one PhysicalNode and one Partition (represented as Exists match elements). The MatchModel of rule 'MapPartition' also has a Partition (Any) match element connected to PhysicalNode and Module (Exists) match elements to represent a PAC. Thus, the rules in the first layer totally overlap if we abstract from the match element types (i.e., Any or Exists). The extension explained in Section 4 combines the rules of the first layer into 


\begin{tabular}{|l|l|l|l|}
\hline Layer & Rule Name & Input Types & Output Types \\
\hline \multirow{3}{*}{1} & MapPhysNode2FiveElements & PhysicalNode & $\begin{array}{l}\text { System, SystemMapping, SoftwareComposition, } \\
\text { CompositionType, EcuInstance }\end{array}$ \\
\cline { 2 - 4 } & MapPartition & Partition & SwcToEcuMapping \\
\cline { 2 - 4 } & MapModule & Module & $\begin{array}{l}\text { SwCompToEcuMapping_component, } \\
\text { ComponentPrototype }\end{array}$ \\
\hline \multirow{2}{*}{2} & MapConnPhysNode2Partition & $\begin{array}{l}\text { PhysicalNode, } \\
\text { Partition }\end{array}$ & $\begin{array}{l}\text { SystemMapping, EcuInstance, } \\
\text { SwcToEcuMapping }\end{array}$ \\
\cline { 2 - 4 } & MapConnPartition2Module & $\begin{array}{l}\text { PhysicalNode, } \\
\text { Partition, } \\
\text { Module }\end{array}$ & $\begin{array}{l}\text { CompositionType, ComponentPrototype, } \\
\text { SwcToEcuMapping, } \\
\text { SwCompToEcuMapping_component }\end{array}$ \\
\hline \multirow{2}{*}{3} & CreatePPortPrototype & Scheduler & PPortPrototype \\
\cline { 2 - 4 } & CreateRPortPrototype & Scheduler & RPortPrototype \\
\hline
\end{tabular}

Table 1. The rules in each layer of the GM-2-AUTOSAR transformation after reimplementing it in DSLTrans, and their input and output types.

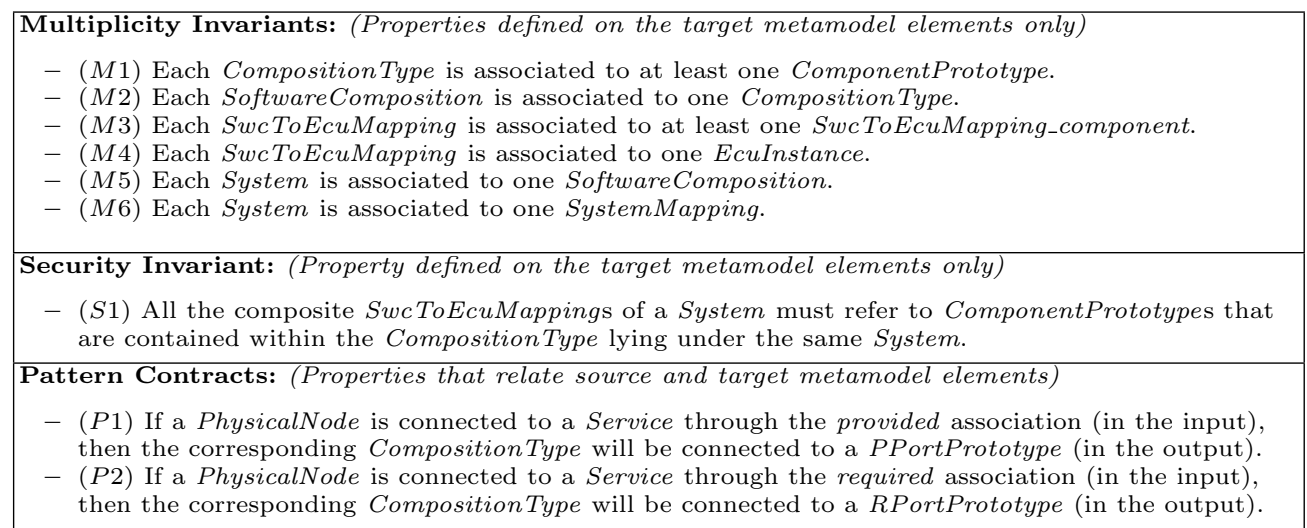

Table 2. Properties of interest for the GM-2-AUTOSAR transformation.

one path condition which simplifies property verification. Partially overlapping rules (Section 4) also occur in layer 2 of our transformation.

\subsection{GM-2-AUTOSAR Model Transformation Properties}

In [17], we stated that properties could be invariants or contracts. Invariants are properties defined on the target metamodel elements only, while contracts relate source and target metamodel elements. Based on these definitions, we further defined four categories of properties in [17]: Multiplicity Invariants, Uniqueness Contracts, Security Invariants, and Pattern Contracts. For each category, we formulated several properties that are summarized in Table 2 and discussed in [17]. We omit Uniqueness Contracts in this study since they require reasoning about attribute values, which is not yet implemented in our property prover.

Multiplicity invariants ensure that the transformation's output preserves the multiplicities in the AUTOSAR metamodel. The security invariant mandates that a System does not refer to a ComponentPrototype that is not allocated in that System. Pattern contracts require that if a pattern of elements is found in the input, then a corresponding pattern of elements must be found in the output.

\subsection{Verifying Properties of the GM-2-AUTOSAR Transformation}

We demonstrate the formulation of pattern contracts (e.g., P1 and P2 in Table 2) in our prover by showing the formulation of $P 1$ in Fig. 12 as an example. $P 1$ 


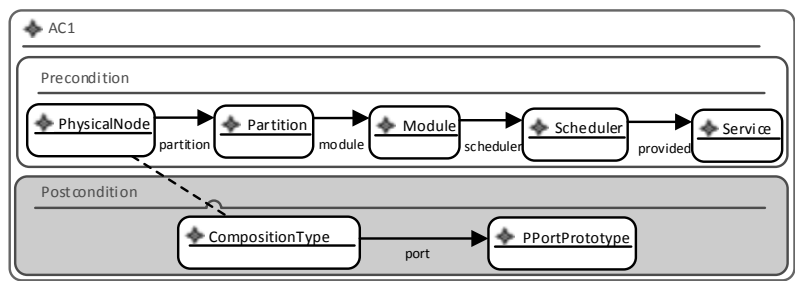

Fig. 12. One Atomic Contract that is used to express property $P 1$.
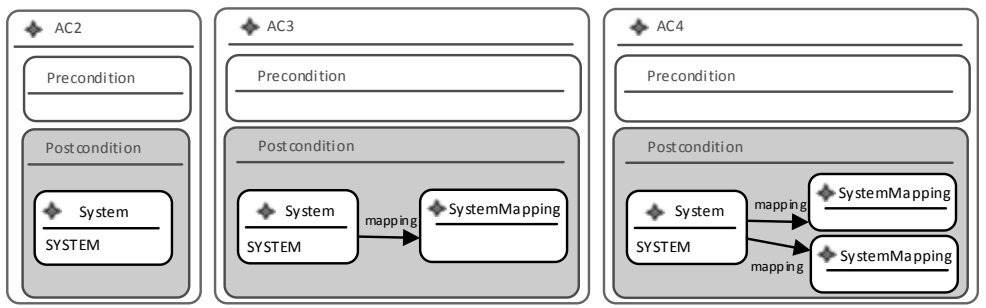

Fig. 13. Three AtomicContracts that are used to express property M6.
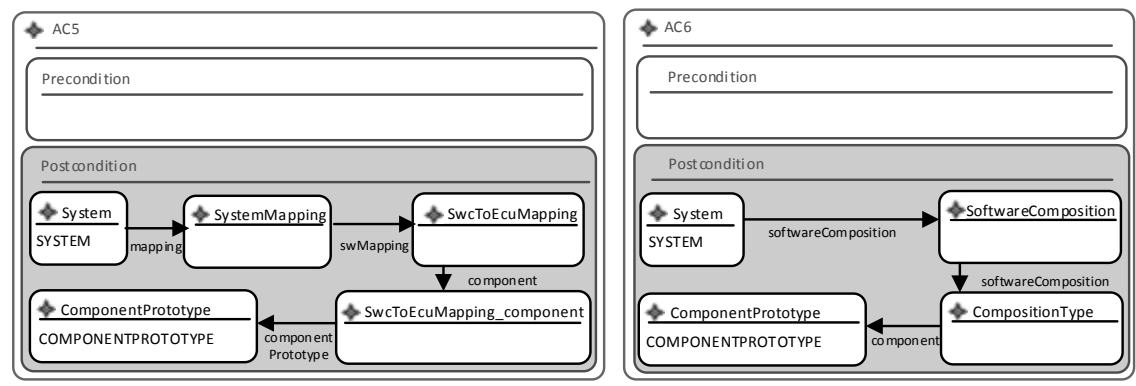

Fig. 14. Two AtomicContracts that are used to express property $S 1$.

mandates that if a PhysicalNode is connected to a Service through the provided association in the input (as in the precondition of Fig. 12), then the corresponding Composition Type will be connected to a PPortPrototype in the output (as in the postcondition). As explained in Section 2, using a traceability link in Fig. 12 mandates that $P 1$ will only match Composition Types that were previously created from PhysicalNodes. We demonstrate the formulation of '1..1' multiplicity invariants (e.g., M2, M4, M5, M6) by showing $M 6$ as an example. $M 6$ ensures that if a System is created in the output, then this System must be connected to one SystemMapping (and not more). Using the AtomicContracts in Fig. 13, M6 can be expressed as $A C 2 \Longrightarrow_{t c}\left(A C 3 \wedge_{t c} \neg_{t c} A C 4\right)$. Variable 'SYSTEM' mandates that if $A C 2$ holds for a specific System, then $A C 3$ should hold and $A C 4$ should not hold for the same System. Changing the former formula to $A C 2 \Longrightarrow_{t c} A C 3$ expresses a 'l.."' multiplicity invariant (e.g., M1, M3). Using the AtomicContracts in Fig. 14, the security invariant $S 1$ can be expressed as $A C 5 \Longrightarrow_{t c} A C 6$. Variables 'SYSTEM' and 'COMPONENTPROTOTYPE' mandate that if $A C 5$ holds for a specific System and ComponentPrototype then AC6 should also hold for the same System and ComponentPrototype. 


\begin{tabular}{|l|c|c|c|c|c|c|c|c|c|}
\hline Property & M1 & M2 & M3 & M4 & M5 & M6 & S1 & P1 & P2 \\
\hline $\begin{array}{l}\text { Verification Time } \\
\text { (our property prover) }\end{array}$ & .013 & .017 & .013 & .017 & .017 & .019 & .017 & .02 & .02 \\
\hline $\begin{array}{l}\text { Verification Time } \\
\text { ([17] at scope 6) }\end{array}$ & 76 & 73.4 & 75 & 75 & 75.5 & 74.5 & 114 & 256 & 251 \\
\hline
\end{tabular}

Table 3. Time taken (in seconds) to verify the properties in Table 2 using our property prover (first row) and using a tool based on constraint solving [17] (second row).

Verification Results: We used our prover to verify the properties in Table 2. The transformation was found to violate $M 1$ and $M 3$, i.e., our prover uncovered the same bugs that we found in the ATL transformation implementation using another tool in [17]. After examining the counter examples (not shown due to space limitations), we identified and fixed the two bugs. The properties were reverified on the updated transformation, and they all returned true. (i.e., our transformation will always satisfy the properties in Table 2).

To assess our prover's performance, we measured the time taken to generate path conditions and to verify the properties (Table 2) of the GM-2-AUTOSAR transformation after fixing the bugs. The prover took on average 0.6 seconds to generate the path conditions. Table 3 (first row) shows the time taken (in seconds) to verify the properties in Table 2 using the generated path conditions. We do not include the time taken for path condition generation in Table 3 since it is performed once for the transformation. The longest time taken to verify a property was 0.02 seconds (P1, P2). Thus, our prover can verify an industrial transformation's properties in a short time. More experiments are needed before we can claim that our prover scales to transformations of varying complexities.

Our property prover and the transformation used in [14] is available at [13]. The industrial transformation is not included for confidentiality reasons.

\section{Discussion}

We discuss the strengths and limitations of our prover by comparing it to a tool that we used to verify the GM-2-AUTOSAR transformation in [17]. The tool we used in [17] verifies ATL (textual) transformations by translating them to a relational representation and then using constraint solvers to prove properties for the translated transformation within a scope (i.e., maximum number of objects per class). In contrast, the prover described in this study verifies DSLTrans (graphical) transformations in their native form (i.e., without translating them to another formalism) using the symbolic transformation executions.

We identify three strengths of our prover in comparison with the tool we used in [17]. First, our prover's verification result holds for all transformation executions and is not limited to a scope. Second, our prover verifies the transformation without translating it to another formalism. Third, our prover verified the properties faster than the tool we used in [17]. Table 3 shows the time taken to verify the properties in Table 2 using our prover (first row) and using the tool in [17] (second row). In Table 3, we only show the results for the smallest scope we used in [17] (i.e., 6). As shown in Table 3, our prover takes significantly shorter time to exhaustively verify the properties, whereas much longer times were needed to verify the same properties in a scope of 6 in [17]. Thus, we claim that our prover scales well in comparison with the tool we used in [17]. 
We identify two limitations of our prover in comparison with the tool we used in [17]. First, although negative application conditions (NACs) are expressible in DSLTrans, our prover cannot verify transformations with rules having NACs. Second, our prover cannot verify properties that reason about attribute values such as the uniqueness contracts (Section 6.2) that we were able to verify in [17]. We are currently working on addressing both limitations in our prover.

\section{Related Work}

We review input-independent verification techniques proposed for (1) textual and (2) graphical transformations, and (3) property languages similar to ours.

(1) Büttner et al. [9] and Cabot et al. [10] translated a transformation and its metamodels into a transformation model and used model finders (e.g., USE Validator) and constraint solvers (e.g., UMLtoCSP) to verify properties. Anastasakis et al. [3] and Baresi and Spoltini [6] translated a transformation into an Alloy model and used the Alloy Analyser to verify the Alloy model within a scope. Troya and Vallecillo [21] translated a transformation into Maude and used Maude's analysis capabilities to verify the transformation. Orejas and Wirsing [15] translated graphs to triple algebras to verify (e.g., using Maude) propositional formula of properties. The study claimed that verifying graph transformations is difficult, and hence the need for the translation to algebra.

(2) Becker et al. [8] verified if a transformation can generate forbidden patterns by checking if the backward application of each rule to each forbidden pattern can produce a valid input, and returns this input as a counterexample. Asztalos et al. [5] implemented a VMTS-based verification tool for in-place transformations. VMTS transformations are expressed as graphical rules scheduled by a control flow graph. The tool assigns conditions to each edge in the control flow graph that are guaranteed to hold for the transformation (when run on any input) at this edge. Assigning conditions is performed by analyzing individual rules to generate their strongest post-conditions and iteratively propagating these conditions using inference rules. Eventually, the final edge in the control flow graph is assigned a condition $p_{\text {final }}$ which will always hold for any input. A property $p$ is then verified by evaluating $p_{\text {final }} \longrightarrow p$. Besides being semi-automated, another limitation of the tool is that a property's verification result may be undecidable either due to (a) the lack of the necessary inference (propagation) rules or (b) the need to collectively analyze the control flow graph instead of analyzing rules separately.

Tools such as Henshin [4] and AGG [20] have the drawback of being inputdependent, i.e., they verify transformations when run on a specific input. Similar to model checkers (e.g., Groove), Henshin [4] generates a state space that simulates all possible transformation executions for a specific input and verifies the generated state space. AGG [20] verifies a property on the input and reverifies it on the output of each rule application. AGG does not check all transformation executions; only the first found execution is verified. AGG, however, performs other types of analysis, e.g., critical pair analysis and graph parsing.

(3) Büttner et al. [9] expressed properties in OCL and verified them using model finders. PaMoMo [11] is a graphical language used to express con- 
tracts and complex properties that manipulate contracts. These properties can be compiled into OCL and injected into any OMG-based transformation implementation (e.g., ATL) for automated verification. The property languages used by Asztalos et al. [5] and AGG [20] are similar to ours; i.e., their graph-based property languages are used in their native graphical format and properties are contracts that can be used to build propositional formulae. The difference is that both studies $[5,20]$ do not introduce a construct equivalent to our free variables which allows contracts in the same formula to refer to a specific element.

Difference between our study and related work: Our study differs from related work in one or more of the following aspects: (i) Verification is performed on an intuitive, graphical language that does not require a mathematical background to be used, e.g., Maude $[21,15]$. (ii) We used our prover to verify a simple and an industrial transformation. (iii) We demonstrated several property kinds that our prover can conclusively verify (unlike [5]) as opposed to verifying specific property kinds, e.g., forbidden patterns [8]. (iv) Verification is based on generating the symbolic executions. (v) We have proved the soundness and completeness of our technique in [12]. Many studies translated a transformation into another formalism and verified properties on the translated transformation $[9,10,3,6$, $21,15]$. Such approaches should prove the soundness of the translated transformation before verifying properties. Moreover, such approaches should translate the verification result back to the original formalism for comprehension. Other studies proposed incomplete techniques that are restricted to a scope [9] or that do not guarantee that the transformation is fault-free, e.g., testing.

While textual property languages (e.g., OCL [9]) have been used for specifying properties, we believe that a graphical property language is useful as more researchers adopt graph transformations due to their intuitive, graphical format. Approaches where graphical properties are translated into a textual formalism (e.g., [11]) have two drawbacks: (a) the soundness of the translation should be proved before verifying the translated properties; (b) the translated properties in [11] cannot be used to automatically verify graphical transformations.

We believe that our graph-based property language (that can be verified without translation to another formalism) and input-independent verification technique advances the state of the art and may encourage users in safety critical domains to use the more intuitive, graph-based transformation languages.

\section{Conclusion and Future Work}

In this study we extended a symbolic model transformation property prover [14, 12] that initially only verified AtomicContracts. The extended prover now verifies AtomicContracts and propositional formulae of AtomicContracts for DSLTrans transformations. We have also extended the original path condition generation algorithm by treating overlapping rules. Further, we demonstrated our property prover on an industrial case study [18]. We showed that the prover is of practical use and features fast property proving times when compared with another prover. We also discussed the strengths and limitations of our prover.

For future work, more experiments on bigger transformations are needed to test the prover's scalability. Moreover, as mentioned in Sections 6.2 and 7, 
we plan to handle NACs and attribute values when generating the set of path conditions to facilitate verifying properties that reason about attribute values.

\section{References}

1. AUTOSAR Consortium. AUTOSAR System Template, http://AUTOSAR.org/index.php? $p=3 \& u p=1 \& u u p=3 \& \quad$ uuup $=3 \& u u u u p=0 \&$ uuuup=0/AUTOSAR_TPS_SystemTemplate.pdf, 2007.

2. M. Amrani, L. Lúcio, G. Selim, B. Combemale, J. Dingel, H. Vangheluwe, Y. Le Traon, and J. R. Cordy. A Tridimensional Approach for Studying the Formal Verification of Model Transformations. In VOLT, pages 921-928, 2012.

3. K. Anastasakis, B. Bordbar, and J. Küster. Analysis of Model Transformations via Alloy. MoDeVVa, pages 47-56, 2007.

4. T. Arendt, E. Biermann, S. Jurack, C. Krause, and G. Taentzer. Henshin: Advanced Concepts and Tools for In-Place EMF Model Transformations. In MoDELS, pages 121-135. Springer, 2010.

5. M. Asztalos, L. Lengyel, and T. Levendovszky. Formal Specification and Analysis of Functional Properties of Graph Rewriting-Based Model Transformation. Software Testing, Verification and Reliability, 23(5):405-435, 2013.

6. L. Baresi and P. Spoletini. On the Use of Alloy to Analyze Graph Transformation Systems. In ICGT, volume 4178 of $L N C S$, pages 306-320, 2006.

7. B. Barroca, L. Lúcio, V. Amaral, R. Félix, and V. Sousa. DSLTrans: A Turing Incomplete Transformation Language. In SLE, pages 296-305. 2011.

8. B. Becker, D. Beyer, H. Giese, F. Klein, and D. Schilling. Symbolic Invariant Verification for Systems with Dynamic Structural Adaptation. In ICSE, 2006.

9. F. Büttner, M. Egea, E. Guerra, and J. De Lara. Checking Model Transformation Refinement. In ICMT, pages 158-173. 2013.

10. J. Cabot, R. Clarisó, E. Guerra, and J. de Lara. Verification and Validation of Declarative Model-to-Model Transformations Through Invariants. Systems and Software, 83(2):283-302, 2010.

11. E. Guerra, J. de Lara, D. Kolovos, and R. Paige. A Visual Specification Language for Model-to-Model Transformations. In $V L / H C C$, pages 119-126. IEEE, 2010.

12. L. Lúcio, B. Oakes, and H. Vangheluwe. A Technique for Symbolically Verifying Properties of Graph-Based Model Transformations. Technical Report SOCS-TR2014.1, McGill U., 2014.

13. L. Lúcio and G. Selim. DSLTrans Property Prover and Example Transformation, http://msdl.cs.mcgill.ca/people/levi/police_station_verification_example.zip.

14. L. Lúcio and H. Vangheluwe. Model Transformations to Verify Model Transformations. In VOLT, 2013.

15. F. Orejas and M. Wirsing. On the Specification and Verification of Model Transformations. In Semantics and algebraic specification, pages 140-161. Springer, 2009.

16. L. A. Rahim and J. Whittle. A Survey of Approaches for Verifying Model Transformations. SoSyM, pages 1-26, 2013.

17. G. Selim, F. Büttner, J. R. Cordy, J. Dingel, and S. Wang. Automated Verification of Model Transformations in the Automotive Industry. In MODELS, 2013.

18. G. Selim, S. Wang, J. R. Cordy, and J. Dingel. Model Transformations for Migrating Legacy Models: An Industrial Case Study. ECMFA, pages 90-101, 2012.

19. E. Syriani and H. Vangheluwe. De-/re-constructing Model Transformation Languages. EASST, 29, 2010.

20. G. Taentzer. AGG: A Graph Transformation Environment for Modeling and Validation of Software. In AGTIVE, pages 446-453. Springer, 2004.

21. J. Troya and A. Vallecillo. A Rewriting Logic Semantics for ATL. JOT, 10:5: 1-29, 2011. 